\title{
Liquid biopsy in the diagnosis of HPV DNA in breast lesions
}

Sabrina De Carolis ${ }^{1,2}$, Alice Pellegrini ${ }^{3}$, Donatella Santini ${ }^{4}$, Claudio Ceccarelli ${ }^{1}$, Antonio De Leo $^{1}$, Federica Alessandrini ${ }^{1}$, Chiara Arienti ${ }^{5}$, Sara Pignatta ${ }^{5}$, Anna Tesei ${ }^{5}$, Vilma Mantovani $^{2}$, Claudio Zamagni ${ }^{6}$, Mario Taffurelli ${ }^{3,7}$, Pasquale Sansone ${ }^{8,9}$, Massimiliano Bonafé ${ }^{1,2}$ \& Monica Cricca*,1

${ }^{1}$ Department of Experimental, Diagnostic \& Specialty Medicine, Alma Mater Studiorum, University of Bologna, 40138, Bologna, Italy

${ }^{2}$ Center of Applied Biomedical Research (CRBA), S. Orsola-Malpighi Hospital, 40138, Bologna, Italy

${ }^{3}$ Department of Women, Children \& Urological Diseases, S. Orsola-Malpighi Hospital, 40138, Bologna, Italy

${ }^{4}$ Operative Unit of Pathology, S. Orsola Malpighi Hospital, 40138, Bologna, Italy

${ }^{5}$ Drug Discovery Unit \& Radiobiology, Biosciences Laboratory, Istituto Scientifico Romagnolo per lo Studio e la Cura dei Tumori (IRST), IRCCS, 47014, Meldola, Italy

${ }^{6}$ Medical Oncology Unit, S. Orsola-Malpighi Hospital, 40138, Bologna, Italy

${ }^{7}$ Department of Medical \& Surgical Sciences, Alma Mater Studiorum, University of Bologna, 40138, Bologna, Italy

${ }^{8}$ Department of Medicine, Memorial Sloan-Kettering Cancer Center, New York, 10021 NY, USA

${ }^{9}$ Department of Pediatrics, Cell \& Developmental Biology, Children's Cancer \& Blood Foundation Laboratories, Weill Cornell

Medical College, New York, 10021 NY, USA

* Author for correspondence: monica.cricca3@unibo.it

\begin{abstract}
Aim: HPV DNA has never been investigated in nipple discharges (ND) and serum-derived extracellular vesicles, although its presence has been reported in ductal lavage fluids and blood specimens. Materials \& methods: We analyzed 50 ND, 22 serum-derived extracellular vesicles as well as 51 pathologic breast tissues for the presence of 16 HPV DNA types. Results: We show that the presence of HPV DNA in the ND is predictive of HPV DNA-positive breast lesions and that HPV DNA is more represented in intraductal papillomas. We also show the presence of HPV DNA in the serum-derived extracellular vesicles. Conclusion: Our data supports the use of liquid biopsy to detect HPV DNA in breast pathology.
\end{abstract}

First draft submitted: 12 July 2017; Accepted for publication: 6 September 2017; Published online: 3 October 2017

Keywords: breast • HPV DNA • liquid biopsy

The presence of HPV DNA in breast cancer has been widely investigated in the last three decades [1], but few data are available on its presence in benign breast lesions, in other words, intraductal papilloma (IP). A plethora of pathogens has been found in triple negative breast cancer, the most aggressive breast cancer phenotype, including mucosal and cutaneous HPVs [2]. The presence of HPV DNA has been reported in biological fluids such as milk and colostrum of lactating women as well as in ductal lavages from women at increased risk of breast cancer [3,4]. The presence of HPV DNA has never been investigated in nipple discharge, a spontaneous or induced biological fluid which is examined to exclude the presence of malignant cells in women with any palpable lump or mammographic abnormalities. Interestingly, HPV DNA has recently been described in the blood of cervical cancer affected women [5]. Recent literature also shows that viral nucleic acids are cargoed by circulating extracellular vesicles (EVs), which are membrane-derived vesicles involved in cell-to-cell communication [6,7].

We first searched for the presence of 16 high-risk mucosal HPV DNA in the virome of breast tissues and their biological fluids, in other words, nipple discharges (ND) and serum-derived EVs, of women with benign or malignant breast cancer lesions. Then, we searched if a noninvasive approach, the liquid biopsy, could be useful in the diagnosis of HPV DNA-positive breast lesions. 


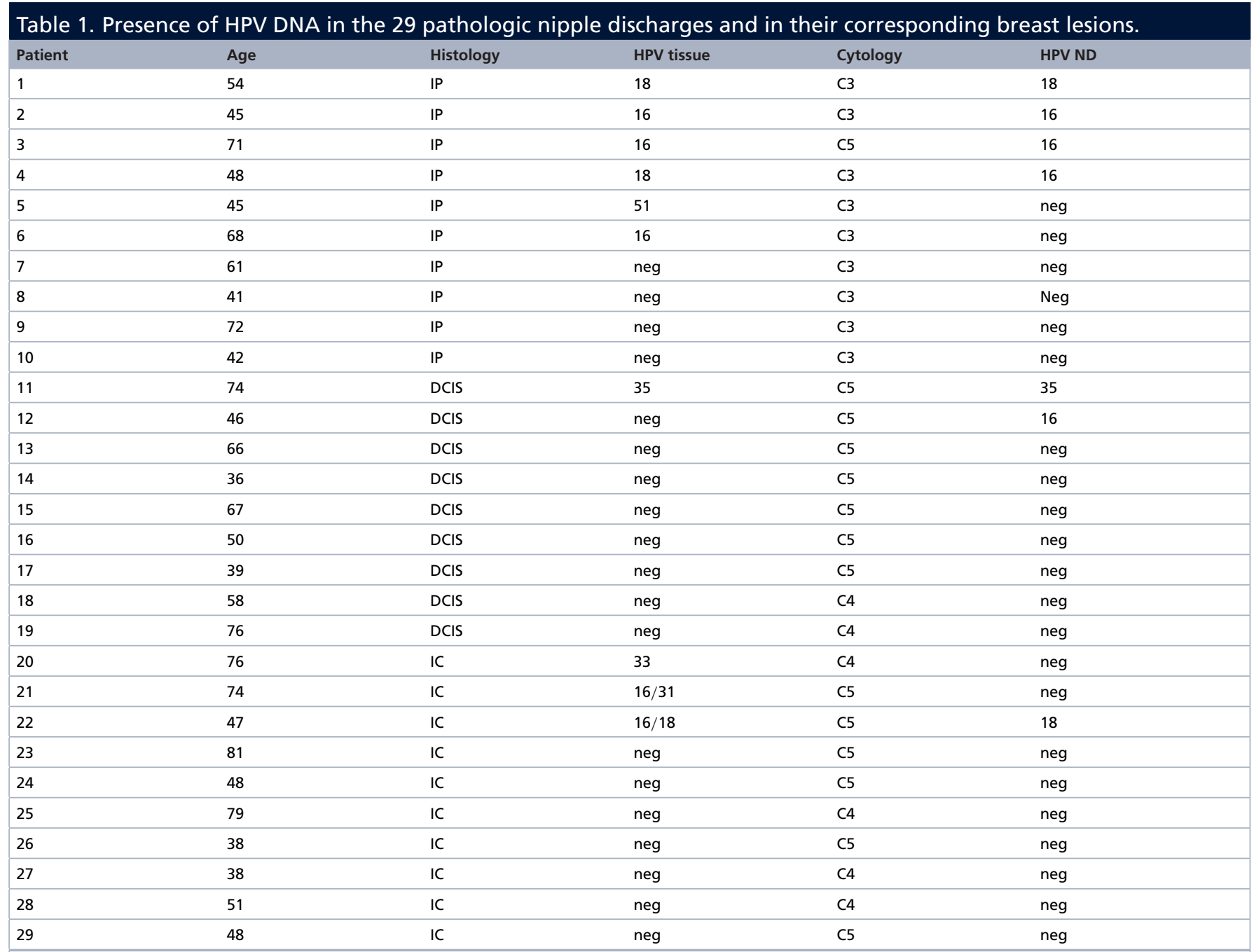

The ND cytology has been categorized as defined by the UK Breast Screening Programme in 1993.

Cytology: C3: Atypia probably benign; C4: Suspicious of malignancy; C5: Malignant; DCIS: Ductal carcinoma in situ; HPV ND: HPV DNA genotype in nipple discharge; HPV tissue: HPV DNA genotype in breast tissue; IC: Invasive carcinoma; IP: Intraductal papilloma.

\section{Materials \& methods \\ Clinical specimens}

A total of 29 pathologic ND and their Formalin Fixed Paraffin Embedded (FFPE) breast tissues were collected at the Breast Unit, Sant'Orsola Malpighi Hospital, Bologna, Italy, from women with abnormal discharges and breast disease. The 29 FFPE breast tissues included 10 IP, 9 ductal carcinoma in situ (DCIS) and 10 invasive carcinoma (Table 1, see samples 1-29). A total of 21 para-physiologic NDs were randomly collected from women without any kind of breast pathology [8] at the Medical Oncology Unit, Sant'Orsola Malpighi Hospital. The study was approved by the Sant'Orsola Malpighi Hospital ethics committee (number 006/2012/U/Tess). All subjects gave written informed consent in accordance with the Declaration of Helsinki. The ND cytological examination has been categorized from C1 to C5 as defined by the UK Breast Screening Programme in 1993.

A total of 22 blood samples and their FFPE breast tissues were collected at the Breast Unit, Sant'Orsola Malpighi Hospital, Bologna, Italy, from women with recently diagnosed breast pathology. The 22 FFPE breast tissues included 2 IP, 1 benign phyllodes tumor, 11 fibroadenoma and 8 DCIS (Table 2, see samples 30-51). This study was approved by the local ethics committee (number 145/2015/U/Sper) and signed, informed consent was obtained from all the patients enrolled. 
Table 2. Presence of HPV DNA in the 22 serum-derived extracellular vesicles and in their corresponding breast lesions.

\begin{tabular}{|c|c|c|c|c|}
\hline Patient & Age & Histology & HPV tissue & HPV EV \\
\hline 30 & 47 & IP & neg & neg \\
\hline 31 & 59 & IP & neg & neg \\
\hline 32 & 45 & BPT & neg & 16 \\
\hline 33 & 30 & FA & neg & neg \\
\hline 34 & 15 & FA & neg & neg \\
\hline 35 & 20 & FA & neg & neg \\
\hline 37 & 27 & FA & neg & neg \\
\hline 38 & 49 & FA & neg & neg \\
\hline 39 & 57 & FA & neg & neg \\
\hline 40 & 47 & FA & neg & neg \\
\hline 41 & 74 & FA & neg & neg \\
\hline 46 & 42 & DCIS & neg & neg \\
\hline 47 & 48 & DCIS & 16 & 16 \\
\hline 48 & 41 & DCIS & neg & neg \\
\hline 49 & 46 & DCIS & neg & neg \\
\hline 50 & 54 & DCIS & neg & neg \\
\hline 51 & 49 & DCIS & 16 & 16 \\
\hline
\end{tabular}

\section{Serum-derived EVs isolation}

EVs were isolated from a $5 \mathrm{ml}$ volume of serum specimen by the gold standard methodology of differential ultracentrifugation [9] (Figure 1A). Isolated serum-derived EVs were resuspended in a $100 \mu$ l volume of phosphate buffer saline. The effective presence of EVs was assessed by Western Blot for Alix expression (Mouse anti-ALIX antibody, clone 3A9, MCA2493, Bio-Rad, Milan Italy) and EVs were counted by NanoSight NS500 (IRST, Meldola, Italy) (Figure 1B). Serum-derived EVs were also DNase-I digested (DNase I RNase-free, Thermo-Fisher, Milan, Italy) prior to nucleic acid extraction (NucleoSpin Tissue XS, Macherey-Nagel, Milan, Italy) to ensure that viral DNA detection pertained to the EVs cargo [10].

\section{Total DNA Isolation \& HPV DNA detection}

Up to a $100 \mu \mathrm{l}$ volume of ND layered on Guthrie cards and total DNA was extracted by NucleoSpin Tissue XS kit (Macherey-Nagel) following the manufacturer's instructions. Total DNA was extracted from three 10- $\mu \mathrm{m}$ thick sections of FFPE breast cancer tissue by NucleoSpin DNA FFPE XS kit (Macherey-Nagel) following the manufacturer's instructions. Finally, total DNA was isolated from a $50 \mu$ l volume of DNAse-I digested serumderived EVs by NucleoSpin Tissue XS (Macherey-Nagel) following the manufacturer's instructions. HPV DNA was assessed by an high-throughput MALDI-TOF mass spectrometry-based method (Mass Array Platform, Agena Bioscience, Hamburg, Germany) as previously described [11]. Briefly, the mass spectrometry-based method is able to simultaneously detect and type 16 HPV DNA types (HPV16, 18, 31, 33, 35, 39, 45, 51, 52, 53, 56, 58, 59, 66, 68 and 73) in a single well. The DNA target sequence is amplified by a multiplex PCR with HPV E6 or E7 gene-specific primers. A primer for primer extension anneals to the amplified product and is extended at its $3^{\prime}$ terminal base for each HPV type. Each product is detected at a specific mass because of the addition of terminating dideoxynucleotides (ddNTPs). This method was able to detect high-risk HPV multiple infections better than conventional PCR assays and was as sensitive to detect up to 100 copies/reaction of the prevalent HPV types (HPV 16 and 18). 
(A)

Differential ultracentrifugation

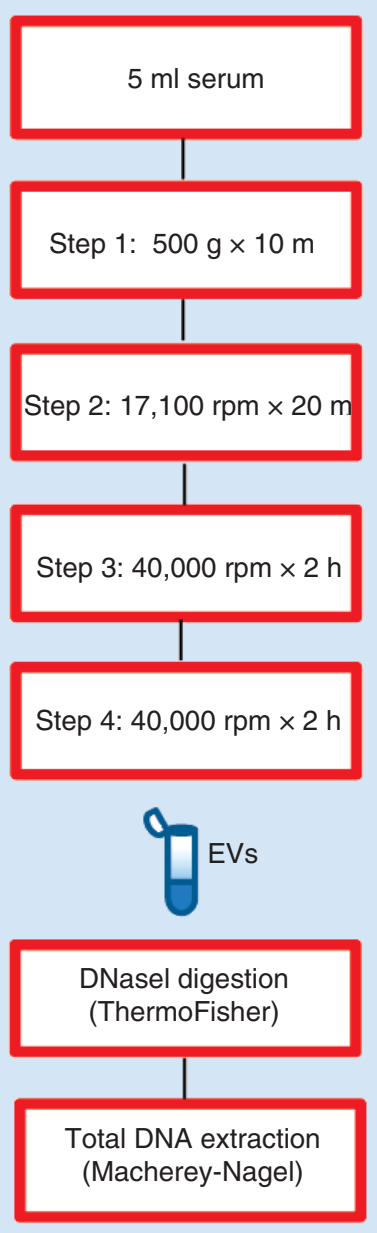

(B)

Sample

\begin{tabular}{|c|c|c|c|c|c|c|c|c|c|}
\hline $\mathrm{k}+$ & 32 & 38 & 40 & 44 & 46 & 47 & 49 & 51 & \\
\hline & & ewa & $\mathrm{na}$ & ene & 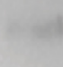 & S & E & $e^{*}$ & $\begin{array}{c}90-100 \mathrm{kDa} \\
\text { Alix }\end{array}$ \\
\hline & 2.7 & 1.8 & 5.5 & 5.2 & 1.7 & 5.8 & 4.9 & 3.9 & $\mathrm{~N} \times 10^{12}$ \\
\hline & 80 & 89 & 79 & 86 & 106 & 79 & 89 & 78 & Mean $(n m)$ \\
\hline
\end{tabular}

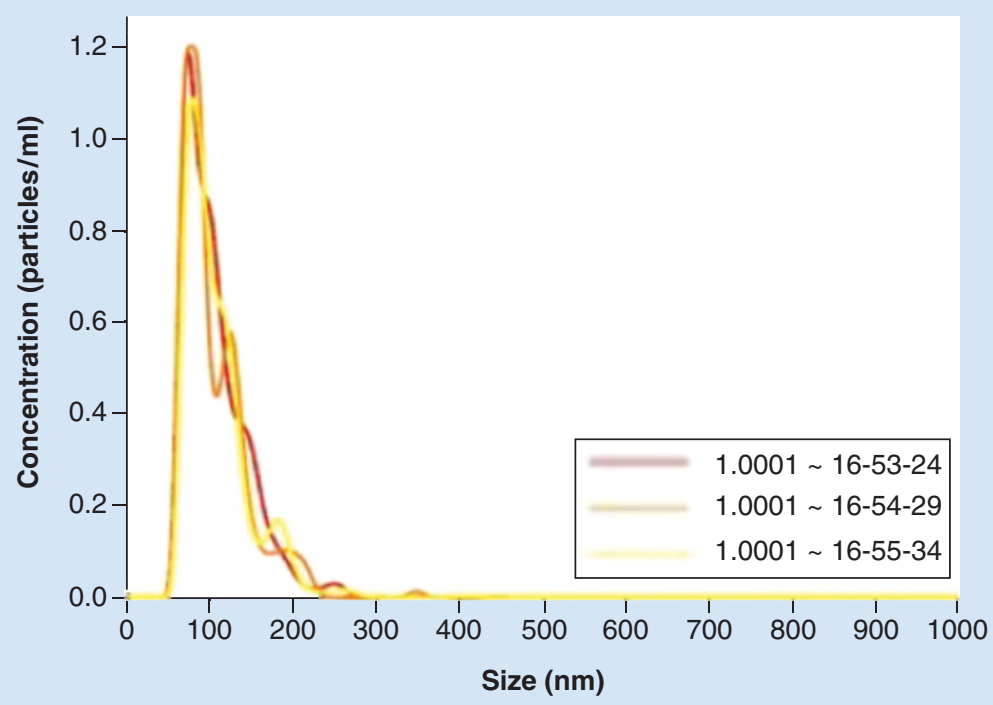

Representative NanoSight plot (Sample 51)

Figure 1. Serum-derived extracellular vesicles isolation and characterization. (A) Serum-derived EVs were isolated by differential ultracentrifugation and DNase-I digested, (B) Western Blot and NanoSight analysis of 8 Alix positive serum-derived EVs (see Table 2) and representative NanoSight plot ( $n=3$ replicates) of sample 51 (see Table 2 ).

EV: Extracellular vesicle.

\section{Results \\ HPV DNA in pathologic \& physiologic ND}

This report was inspired by an observation made on a 54-year-old Caucasian woman suffering from an abundant, spontaneous and serous ND of the left breast, without any palpable lump. The cytological examination of ND showed the presence of atypical and inflammatory, red blood cells (cytology grade C3). Galactography revealed a main duct ectasia with an intraductal filling defect (Figure 2A). The galactoforectomy (Figure 2B) showed an IP (Figure 2C) without any sign of malignancy. By Chromogenic in situ Hybridization (ZytoFastPlus CISH Implementation kit HRP-DAB, Bio-Optica and HPV16/18 Digoxigenin labeled probes) we found the presence of HPV DNA 16/18 in the IP tissue (Figure 2D). HPV DNA was then assessed in both ND and IP by PCR assays targeting the HPV E6 gene of HPV16 and 18 (HPV16 forward primer, nt positions 206-226, 5' - CAACAGTTACTGCGACGTGAG-3', HPV16 reverse primer, nt positions 534-554, 5'GCTGGGTTTCTCTACGTGTTC-3', NCBI reference sequence: NC_001526.4; HPV18 forward primer, nt positions 156-177, 5'-TGCACGGAACTGAACACTTCAC-3', HPV18 reverse primer, nt positions 480-501, 5' GCCCAGCTATGTTGTGAAATCG-3' ${ }^{\prime}$, NCBI reference sequence: NC_001357.1). PCR reaction was performed 

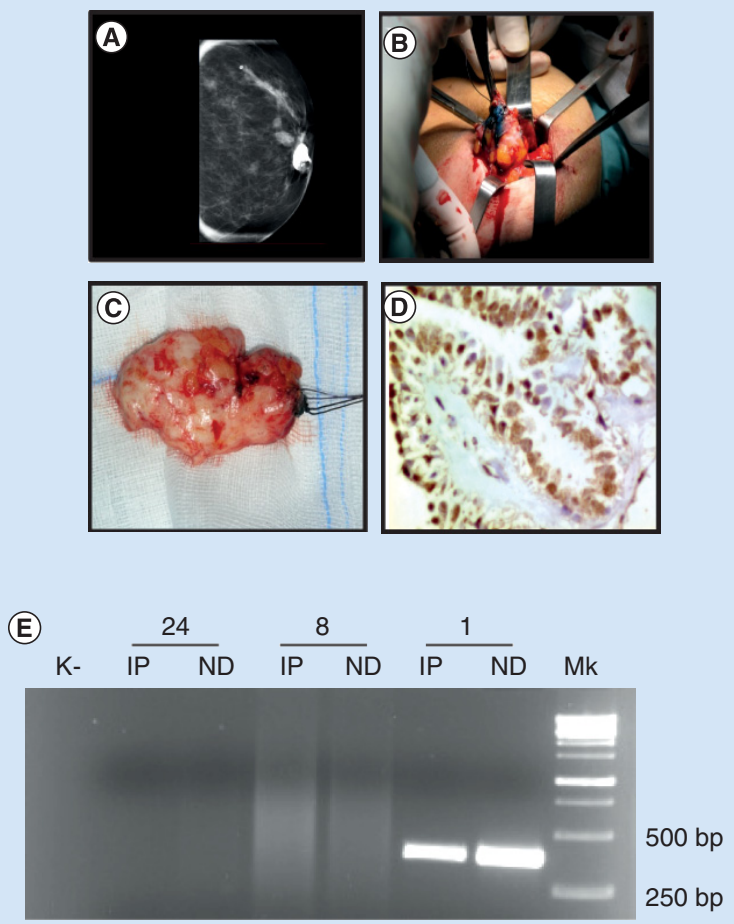

(F)

Human papillomavirus type 18 complete sequence

Sequence ID: AY262282.1 Length: 7857 Number of matches: 1

\begin{tabular}{lclll} 
Range 1: 212 to 501 & \multicolumn{2}{l}{ GenBank Graphics } & Next match Previou \\
\hline Score & Expect & Identities & Gaps & Strand \\
524 bits (580) & $5 e-145$ & $290 / 290(100 \%)$ & $0 / 290(0 \%)$ & Plus/Plus
\end{tabular}

Query 1 GACAGTATTGGAACTTACAGAGGTATTTGAATTTGCATTTAAAGATTTATTTGTGGTGTA 60

Sbjct 212 GACAGTATTGGAACTACAGAGGTATTGATTIGCATTTAAAGATTATTTGTGGTGTA 271

Query 61 TAGAGACAGTATACOGCATGCTGCATGCCATAAATGTATAGATTTTTATTCTAGAATTAG 120

Sbjct 272 TAGAGACAGTATACOGCATGCTGCATGCCATAAATGTATAGAT TTTATTCTAGAATTAG 331

Query 121 AGAATTAAGACATTATTCAGACTCTGTGTATGGAGACACATTGGAAAAACTAACTAACAC 180

Sbjct 332 AGAATTAAGACATTATTCAGACTCTGTGTATGGAGACACATTGGAAAACTAACTAACAC 391

Query 181 TGGGTTATACAATTTATTAATAAGGTGOCTGCGGTGOCAGAAACCGTTGAATCCAGCAGA 240

sbjct 392 TGGGTATACAATTTATTAATAAGGTGCCTGCGGTGOCAGAAACCGTTGAATCCAGCAGA 451

Query 241 AAAACTTAGACACCTTAATGAAAAACGACGATTTCACAACATAGCTGGGC 290

Sbjct 452 AAAACTTAGACACCTAATGAAAACGACGATTTCACAACATAGCTGGGC 501

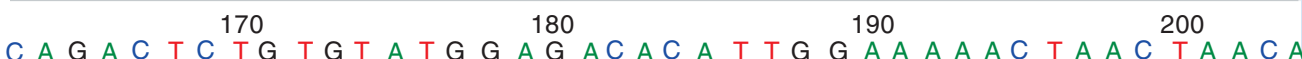

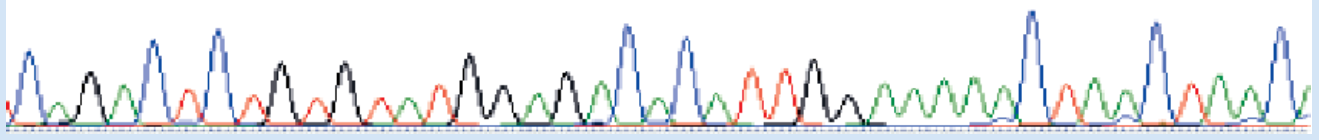

Figure 2. Case report of a woman with the same HPV strain in both intraductal papilloma and nipple discharge. (A) Galactography which shows an intraductal filling defect, (B \& C) Duct excision procedure (galactoforectomy) and the removed IP, (D) Presence of HPV DNA in the papilloma tissue as revealed by chromogenic in situ hybridization. (E) The electrophoresis shows the same HPV strain (HPV18) in ND and IP (see sample 1, Table 1), (F) First result of the nucleotide blast search using as query the ND nucleotide sequence. IP: Intraductal papilloma; ND: Nipple discharge. 
with $2 \mathrm{mM}$ of $\mathrm{MgCl}_{2}$ and for 35 cycles at $94^{\circ} \mathrm{C}$ for $30 \mathrm{~s}, 58^{\circ} \mathrm{C}$ for $30 \mathrm{~s}$ and $72^{\circ} \mathrm{C}$ for $30 \mathrm{~s}$. The electrophoresis showed the same HPV strain (HPV18) in ND and IP (Figure 2E, sample 1, see also Table 1). The sequencing results and nucleotide blast search are showed in Figure 2F.

Prompted by these results, 29 FFPE breast tissues, whose pathologic ND were also available, were examined for the presence of HPV DNA. We found a greater prevalence of HPV DNA in IP than in malignant and early malignant lesions (Fisher test; $\mathrm{p}=0.04,60$ vs $21 \%$, respectively). Furthermore, we found eight single infections (three HPV16, two HPV18, one HPV33, one HPV 51 and one HPV 53) and three multiple infections (HPV16/31 and HPV16/18). We then assessed their corresponding 29 pathologic ND (Table 1) and we compared them with 22 physiologic ND, which were normal discharges, not associated with any recognizable breast disease. Higher prevalence of HPV DNA was detected in pathologic ND versus physiologic ones (Fisher test; $p=0.054,7$ out of $29,24 \%$ vs 1 out of $22,5 \%$ ) and HPV16 was the main HPV type. Moreover, HPV DNA was not significantly associated with the cytology grade (Fisher test; $\mathrm{p}=0.26$, C3 vs C4/5). Finally, the presence of HPV DNA in pathologic NDs was indicative of HPV DNA presence in $83 \%$ of breast tissues (McNemar test for discordant values; $\mathrm{p}=0.26$ ). In four out of six women (one IC and three IP) the same HPV type was harbored in both ND and breast tissue (Table 1). The notion that HPV DNA can be found in breast fluids has previously been reported in colostrum, milk and ductal lavage [3,4]. Therefore, our data confirm that HPV is present in the breast tissue but does not seem strictly related to malignancy per se, as it is more represented in IPs. In this regard, an IP with multiple high-risk HPV infection was already reported in literature [12].

\section{HPV DNA in serum-derived EVs}

Recent literature reports that viral genomes are cargoed in the body fluids by EVs [6,7]. Although viremia is absent in HPV related-diseases, HPV DNA has already been reported in the blood [5]. We enrolled 22 women with breast disease (Table 2) and we investigated the presence of HPV DNA in their serum-derived EVs by the mass spectrometry based method. We found 2/8 (25\%) HPV DNA positive serum-derived EVs among DCIS affected patients and 1/14 (7\%) HPV DNA positive serum-derived EVs among benign breast disease affected women (Table 2). Then we searched for HPV DNA in the corresponding breast tissues and we found that 3 out of 22 were HPV DNA positive (two DCIS and one fibroadenoma) (Table 2). Notably, there was a complete correspondence between HPV DNA in DCIS tissues and serum-derived EVs. Thus, our data show that circulating HPV DNA is at least in part cargoed inside the EVs of breast disease affected women.

\section{Discussion}

We showed that the detection of HPV DNA in pathologic NDs is predictive of its presence in breast lesions in $83 \%$ of the cases. The presence of HPV DNA in NDs confirms that breast tissues harbor mucosal high-risk HPV as constituents of the local virome. These data are in agreement with recent literature also showing that cutaneous HPVs take part in it [2]. As HPV DNA is more represented in IP, its presence seems not to be strictly related to malignancy. However, this fact does not exclude that HPV DNA may influence breast cancer aggressiveness, since we [Unpublished DATA] and others [13,14] documented a higher prevalence of HPV DNA in more aggressive breast cancer phenotypes. In this regard, as the presence of HPV oncogenic proteins is higher in precancerous breast lesions than in invasive ones [15], breast carcinogenesis may be affected by still unrecognized mechanisms related to HPV infection. Moreover, considering the recent report on circulating HPV DNA in cervical cancer affected women [5], HPV DNA assessment in EVs may offer the unprecedented opportunity to expand the field of liquid biopsy in the diagnosis of HPV DNA-positive breast lesions. Finally, since a plethora of human cancers have been associated with HPV DNA in extra-genital body sites [16,17], circulating EVs might represent an alternative way to viremia for delivering HPV DNA in noncanonical sites of infection.

\section{Acknowledgements}

We would like to thank C Pizzirani for technical support, $\vee$ Cocchi for nipple discharge examination and D Sarnacchiaro for the English revision. 


\section{Summary points}

- The presence of HPV DNA has been associated with breast cancer since 1992. Few studies have analyzed its presence in biological fluids such as ductal lavages and blood specimens. In this study, we analyzed the presence of 16 high-risk mucosal HPV DNA by a mass spectrometry-based method in nipple discharges (NDs) and serum-derived extracellular vesicles (EVs) as well as in their corresponding benign and malignant breast tissues.

- First, we report the presence of the same HPV type, HPV18, in both the ND and the papilloma of a woman suffering from spontaneous serous ND and without any palpable lump.

- We show that the presence of HPV DNA in NDs was predictive of the presence of HPV DNA in breast lesions (McNemar test for discordant values, $\mathrm{p}=0.26$ ) and we report a major prevalence of HPV DNA in intraductal papilloma lesions than in malignant ones (Fisher test; $p=0.04$ ).

- We also demonstrate that HPV DNA circulates in the serum-derived EVs and that two out of the three HPV DNA positive EVs were from two women with HPV DNA positive ductal carcinoma in situ.

- In conclusion, HPV DNA assessment in biological fluids may offer the unprecedented opportunity to expand the field of liquid biopsy in the diagnosis of HPV DNA-positive breast lesions.

any organization or entity with a financial interest in or financial conflict with the subject matter or materials discussed in the manuscript apart from those disclosed.

No writing assistance was utilized in the production of this manuscript.

\section{Ethical conduct of research}

The authors state that they have obtained appropriate institutional review board approval or have followed the principles outlined in the Declaration of Helsinki for all human or animal experimental investigations. In addition, for investigations involving human subjects, informed consent has been obtained from the participants involved.

\section{Open access}

This work is licensed under the Attribution-NonCommercial-NoDerivatives 4.0 Unported License. To view a copy of this license, visit http://creativecommons.org/licenses/by-nc-nd/4.0/

\section{References}

1. Bae JM, Kim EH. Human papillomavirus infection and risk of breast cancer: a meta-analysis of case-control studies. Infect. Agent Cancer. 14(11), 14 (2016).

2. Banerjee S, Wei Z, Tan F et al. Distinct microbiological signatures associated with triple negative breast cancer. Sci. Rep. 15(5), 15162 (2015).

3. Cazzaniga M, Gheit T, Casadio C et al. Analysis of the presence of cutaneous and mucosal papillomavirus types in ductal lavage fluid: milk and colostrum to evaluate its role in breast carcinogenesis. Breast Cancer Res. Treat. 114(3), 599-605 (2009).

4. Glenn WK, Whitaker NJ, Lawson JS. High risk human papillomavirus and Epstein Barr virus in human breast milk. BMC Res. Notes 1(5), 477 (2012).

5. Jeannot E, Becette V, Campitelli M et al. Circulating human papillomavirus DNA detected using droplet digital PCR in the serum of patients diagnosed with early stage human papillomavirus-associated invasive carcinoma. J. Pathol. Clin. Res. 2(4), 201-209 (2016).

6. Chahar HS, Bao X, Casola A. Exosomes and their role in the life cycle and pathogenesis of RNA viruses. Viruses 7(6), 3204-3225 (2015).

7. Schorey JS, Cheng Y, Singh PP, Smith VL. Exosomes and other extracellular vesicles in host-pathogen interactions. EMBO Rep. 16(1), 24-43 (2015).

8. Montroni I, Santini D, Zucchini G et al. Nipple discharge: is its significance as a risk factor for breast cancer fully understood? Observational study including 915 consecutive patients who underwent selective duct excision. Breast Cancer Res. Treat. 123(3), 895-900 (2010).

9. Théry C, Amigorena S, Raposo G, Clayton A. Isolation and characterization of exosomes from cell culture supernatants and biological fluids. Curr. Protoc. Cell Biol. Chapter 3, Unit 3.22 (2006).

10. Thakur BK, Zhang H, Becker A et al. Double-stranded DNA in exosomes: a novel biomarker in cancer detection. Cell Res. 24(6), 766-769 (2014).

11. Cricca M, Marasco E, Alessandrini F et al. High-throughput genotyping of high-risk human papillomavirus by MALDI-TOF mass spectrometry-based method. New Microbiol. 38(2), 211-223 (2015).

12. Khammapirad T, Prueksadee J, Diaz-Arrastia C et al. Intraductal papilloma of the breast in association with preoncogenic gene of breast cancer. Asian Pac. J. Trop. Biomed. 1(2), 161-163 (2011). 
13. Corbex M, Bouzbid S, Traverse-Glehen A et al. Prevalence of papillomaviruses: polyomaviruses and herpesviruses in triple-negative and inflammatory breast tumors from algeria compared with other types of breast cancer tumors. PLoS ONE 9(12), e114559 (2014).

14. Piana AF, Sotgiu G, Muroni MR, Cossu-Rocca P, Castiglia P, De Miglio MR. HPV infection and triple-negative breast cancers: an Italian case-control study. Virol. J. 21(11), 190 (2014).

15. Lawson JS, Glenn WK, Whitaker NJ. Human papilloma viruses and breast cancer assessment of causality. Front. Oncol. 29(6), 207 (2016)

16. Zur Hausen H. Papillomaviruses in the causation of human cancers - a brief historical account. Virology 384(2), 260-265 (2009).

17. Vidone M, Alessandrini F, Marucci G et al. Evidence of association of human papillomavirus with prognosis worsening in glioblastoma multiforme. Neuro Oncol. 16(2), 298-302 (2014). 